\title{
Dermatofibrosarcoma Protuberans with Myoid Differentiation
}

National Cancer Institute

\section{Source}

National Cancer Institute. Dermatofibrosarcoma Protuberans with Myoid Differentiation. NCl Thesaurus. Code C38105.

A morphologic variant of dermatofibrosarcoma protuberans characterized by the presence of areas of myofibroblastic differentiation. 